Article

\title{
Preparation and Antioxidant Activity of Ethyl-Linked Anthocyanin-Flavanol Pigments from Model Wine Solutions
}

\author{
Lingxi Li ${ }^{1,2}$, Minna Zhang ${ }^{3}$, Shuting Zhang ${ }^{2}$, Yan Cui ${ }^{2}$ and Baoshan Sun ${ }^{2,4, *}$ \\ 1 School of Pharmacy, Shenyang Pharmaceutical University, Shenyang 110016, China; lingxilee@163.com \\ 2 School of Functional Food and Wine, Shenyang Pharmaceutical University, Shenyang 110016, China; \\ zhangshuting@syphu.edu.cn (S.Z.); cuiyan@syphu.edu.cn (Y.C.) \\ 3 School of Traditional Chinese Materia Medica, Shenyang Pharmaceutical University, Shenyang 110016, \\ China; 107000305@syphu.edu.cn \\ 4 Pólo Dois Portos, Instituto National de Investigação Agrária e Veterinária, I.P., Quinta da Almoinha, \\ Dois Portos 2565-191, Portugal \\ * Correspondence: baoshan.sun@gmail.com; Tel: + 86-244-352-0301
}

Received: 2 March 2018; Accepted: 24 April 2018; Published: 3 May 2018

\begin{abstract}
Anthocyanin-flavanol pigments, formed during red wine fermentation and storage by condensation reactions between anthocyanins and flavanols (monomers, oligomers, and polymers), are one of the major groups of polyphenols in aged red wine. However, knowledge of their biological activities is lacking. This is probably due to the structural diversity and complexity of these molecules, which makes the large-scale separation and isolation of the individual compounds very difficult, thus restricting their further study. In this study, anthocyanins (i.e., malvidin-3-glucoside, cyanidin-3-glucoside, and peonidin-3-glucoside) and (-)-epicatechin were first isolated at a preparative scale by high-speed counter-current chromatography. The condensation reaction between each of the isolated anthocyanins and (-)-epicatechin, mediated by acetaldehyde, was conducted in model wine solutions to obtain ethyl-linked anthocyanin-flavanol pigments. The effects of $\mathrm{pH}$, molar ratio, and temperature on the reaction rate were investigated, and the reaction conditions of $\mathrm{pH}$ 1.7, molar ratio 1:6:10 (anthocyanin/(-)-epicatechin/acetaldehyde), and reaction temperature of $35{ }^{\circ} \mathrm{C}$ were identified as optimal for conversion of anthocyanins to ethyl-linked anthocyanin-flavanol pigments. Six ethyl-linked anthocyanin-flavanol pigments were isolated in larger quantities and collected under optimal reaction conditions, and their chemical structures were identified by HPLC-QTOF-MS and ECD analyses. Furthermore, DPPH, ABTS, and FRAP assays indicate that ethyl-linked anthocyanin-flavanol pigments show stronger antioxidant activities than their precursor anthocyanins.
\end{abstract}

Keywords: anthocyanin; antioxidant activity; ethyl-linked anthocyanin-flavanol pigment

\section{Introduction}

Grape and wine polyphenols have attracted considerable attention among the international scientific community during the last four decades, not only for their contributions to the quality of wines, including sensory properties (color, flavor, astringency, and bitterness) [1-4] and aging behavior, but especially for their potential beneficial health effects related to their protective action against coronary heart disease and oxygen free-radical scavenger capacity [5-11].

Red grape polyphenols essentially consist of anthocyanins (mainly malvidin-3-glucoside, cyanidin-3-glucoside, and peonidin-3-glucoside), flavanols (monomeric (+)-catechin and (-)-epicatechin, and their oligomers and polymers), and small amounts of other phenolics 
such as phenolic acids, resveratrol and its derivatives, flavonols, flavanonols, and flavones. Red wine polyphenols include both grape polyphenols (anthocyanins and flavanols) and new phenolic products formed from them during the winemaking process (anthocyanin-derived pigments and polymeric flavanols) [12]. Anthocyanin-derived pigments are formed essentially by labile anthocyanins and tend to react with flavanols during the wine fermentation and aging processes $[13,14]$.

Studies have shown that anthocyanin contents increase sharply after crushing the grapes during winemaking and reach their maximum within 2 to 3 days of maceration/alcoholic fermentation, but then decrease gradually $[15,16]$. In the late period of alcohol fermentation, anthocyanins react with flavanols to form anthocyanin-flavanol pigments [17,18].

There are two acknowledged mechanisms for the formation of new anthocyanin-flavanol pigments $[1,19,20]$. One is the direct condensation reaction between anthocyanins (A) and flavanols (F), which forms the direct condensation products, including $\mathrm{A}^{+}-\mathrm{F}$ or $\mathrm{F}-\mathrm{A}^{+}$adduct [21]. The other is the indirect condensation reaction between anthocyanins and flavanols mediated by an aldehyde linkage $[22,23]$. These products arise through nucleophilic addition of the flavanol to a protonated acetaldehyde, giving a new carbocation intermediate which undergoes nucleophilic addition of an anthocyanin in the hemiketal form to ultimately produce ethyl-linked F-Et-A adducts [24]. Indirect condensation reaction occurs quickly and is the main and common polymerization reaction in wine $[17,20,25]$.

Anthocyanin-flavanol pigments from the condensation reaction between anthocyanins and flavanols play an important role in wine color stabilization and are the major contributor to the color of aged wine [26]. The color of young red wine is bright and usually appears violet or ruby red, which is generated solely from anthocyanins. With the formation of anthocyanin-flavanol pigments, red wine acquires an increasingly deep color, appearing brick-red and even red-brown [27].

Condensation reactions lead to the rapid decrease of anthocyanin content, but the color of red wine remains relatively stable because anthocyanin-flavanol pigments are better able to retain their color than anthocyanins under the same $\mathrm{pH}$ and $\mathrm{SO}_{2}$ conditions [28]. Acetaldehyde, as a byproduct of yeast fermentation that forms through the oxidation of ethanol, can increase the condensation reaction rate of anthocyanins $[23,29]$.

Earlier work reported that pigmented complex in red wine account for up to $50 \%$ of color density within the first year, and up to $90 \%$ after wine aging [30]. Eglinton et al. [18] indicated that pigmented polymers in red wine form during the period of alcohol fermentation. Sun and Spranger [31] reported that during the storage of young red wines, the concentrations of anthocyanins and flavanols decrease significantly while total polyphenols remain stable, suggesting that the majority of anthocyanins and flavanols are transformed to their condensed forms. Quantitatively, anthocyanin-flavanol pigments are one of the major groups of polyphenols in aged red wines [12].

It is well known that moderate consumption of red wine may have beneficial effects, such as protection against certain cancers, improved mental health, and enhanced heart health [32-35], and that the key compounds responsible for these beneficial effects are polyphenols [11,36,37]. Nevertheless, the available data on the biological activities of red wine polyphenols are limited to those of anthocyanins and flavanols [7,38], resveratrol [39], phenolic acids [40], and flavonols [41]. Indeed, anthocyanins and flavanols are the two major groups of polyphenols in red grapes and in some very young red wines but are present at very low concentrations in aged red wines. For the latter, the major polyphenols (from a quantitative perspective) are polymeric polyphenols, including direct and indirect condensation products between anthocyanins and flavanols [42].

However, little is known about the biological properties of the anthocyanin-derived pigments in red wine. This is probably due to the structural diversity and complexity of these compounds, which makes their isolation and further study highly challenging. The objective of the present study was to verify the in vitro antioxidant activity of the ethyl-linked anthocyanin-flavanol pigments formed by indirect condensation reactions between anthocyanins and flavanol that commonly occur during red wine making and storage. For this purpose, we first prepared at large scale the major wine anthocyanins 
(malvidin-3-glucoside, cyanidin-3-glucoside, and peonidin-3-glucoside), and (-)-epicatechin by high-speed counter-current chromatography (HSCCC) and then performed condensation reactions between anthocyanins and (-)-epicatechin, mediated by acetaldehyde, in a model wine solution. The antioxidant activities of the different ethyl-linked anthocyanin-flavanol pigments isolated under optimized reaction conditions were verified by DPPH, ABTS, and FRAP assays.

\section{Results and Discussion}

\subsection{HSCCC Separation of Anthocyanins and (-)-Epicatechin}

Under optimal conditions, three anthocyanins were successfully separated using HSCCC. The yields of malvidin-3-glucoside, peonidin-3-glucoside, and cyanidin-3-glucoside were $12.12 \mathrm{mg}$ (purity 92.74\%), $11.57 \mathrm{mg}$ (purity 91.21\%), and $40.33 \mathrm{mg}$ (purity 94.08\%), respectively. By further purification, the purities of malvidin-3-glucoside, peonidin-3-glucoside, and cyanidin-3-glucoside reached $98.43 \%, 97.29 \%$, and $98.9 \%$, respectively.

By one-step HSCCC separation of flavanol under optimized conditions, the product was predominantly (-)-epicatechin, yielding up to $61.28 \mathrm{mg}$, and $>95 \%$ purity following purification.

\subsection{Dynamic Monitoring of Condensation Reactions between Anthocyanins and (-)-Epicatechin}

HPLC-DAD was used for monitoring the changes in concentrations of anthocyanins and ethyl-linked anthocyanin-flavanol pigments during the reaction period. The HPLC chromatograms of the condensation reaction solutions between cyanidin-3-glucoside/malvidin-3-glucoside/peonidin-3 -glucoside and (-)-epicatechin at 0,2, 224, and $336 \mathrm{~h}$ in the presence of acetaldehyde at $\mathrm{pH} 1.7$ are presented in Figure 1. Peaks 1 and 2 were the main products of each reaction solution. However, with the reaction process, they tended to break down to form a series of byproducts. The structures of the six main products are illustrated as follows. Table 1 presents the effects of $\mathrm{pH}$, molar ratio, and temperature on the reactivity of anthocyanins towards (-)-epicatechin in the presence of acetaldehyde. The influence of $\mathrm{pH}$ on reaction rate constant $K$ was significant, with lower $\mathrm{pH}$ of reaction medium associated with higher reaction rate. This result was consistent with previous studies $[20,22]$ and might be related to the activity of anthocyanin in the flavylium form under acidic conditions [43]. At the same temperature, the rate constants were 0.0497, 0.0565, and 0.0919 for the reaction between malvidin-3-glucoside and (-)-epicatechin at molar ratios 1:1, 1:3, and $1: 6$, respectively, at $\mathrm{pH} 1.7$, as compared with $0.0028,0.0065$, and 0.009 for the reaction between malvidin-3-glucoside and (-)-epicatechin at molar ratios 1:1, 1:3, and 1:6, respectively, at pH 3.2. These results indicate that increased (-)-epicatechin molar concentration leads to an increased reaction rate. Since the $\mathrm{pH}$ of the wine ranged from 3.2 to 3.5, theoretically, the rate constants of reactions between anthocyanins and (-)-epicatechin in the wine solution are closer to the values obtained

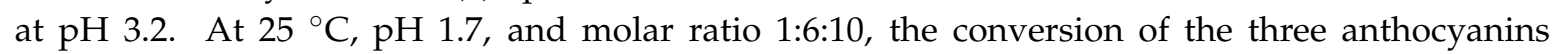
to ethyl-linked anthocyanin-flavanol pigments showed differing reaction rate constants, in this sequence: peonidin-3-glucoside $>$ cyanidin-3-glucoside $>$ malvidin-3-glucoside. However, at $\mathrm{pH}$ 3.2 representing the $\mathrm{pH}$ of red wine, peonidin-3-glucoside showed the highest rate constant and cyanidin-3-glucoside the lowest. The condensation reaction might be influenced by the structure of anthocyanins [44]; the methylation at C-3 position of peonidin-3-glucoside and malvidin-3-glucoside might affect the reaction rate. Meanwhile, the findings for the reaction rate constant $K$ might represent the differing contents and stabilities of these three anthocyanins in wine. Peonidin-3-glucoside and malvidin-3-glucoside, which had faster reaction rates, could rapidly react with flavanol and become polymerized into more anthocyanin-flavanol pigments in a stable form in wine, thus contributing more to the wine color. In contrast, cyanidin-3-glucoside, which showed a slower reaction rate, might be easily oxidized and lead to degradation due to the complex variations in conditions during wine aging, therefore contributing less to the wine color than peonidin-3-glucoside and cyanidin-3-glucoside. Similar conclusions were noted by previous researchers [45]. In conclusion, lowering the $\mathrm{pH}$ of the 
reaction medium, increasing the reaction temperature, and increasing the molar ratio of the reactants could increase the reaction rate and produce higher yields of the ethyl-linked anthocyanin-flavanol pigments. Although the reaction rate peaked at $40{ }^{\circ} \mathrm{C}$, the formation and conversion of the main ethyl-linked anthocyanin-flavanol pigments products were both too rapid, thereby hindering their collection. Consequently, reaction conditions $\mathrm{pH} 1.7$, molar ratio 1:6:10, and temperature $35^{\circ} \mathrm{C}$ were optimal for large-scale preparation of ethyl-linked anthocyanin-flavanol pigments.
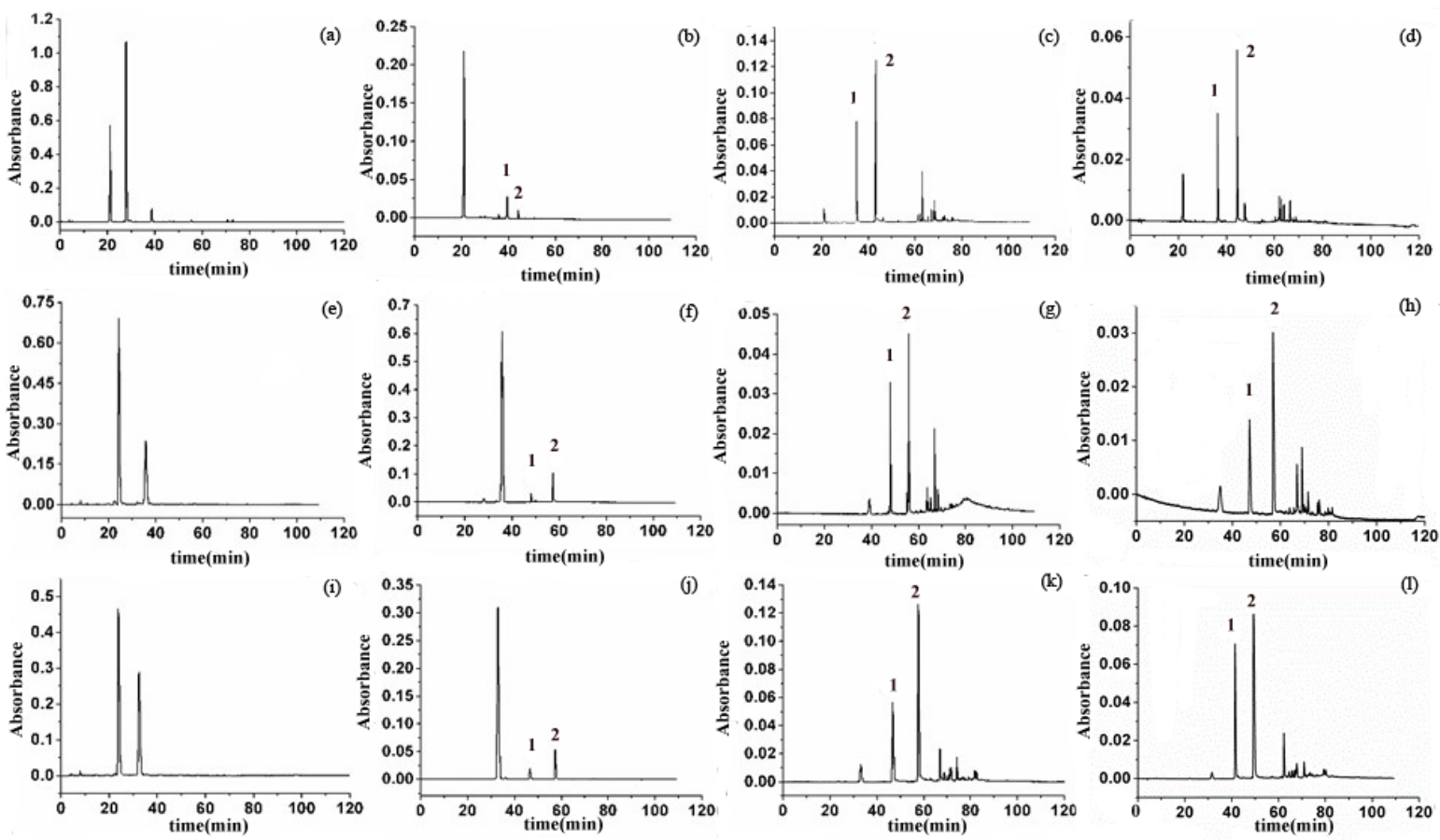

Figure 1. Chromatograms of HPLC analysis of the reaction between cyanidin-3-glucoside and

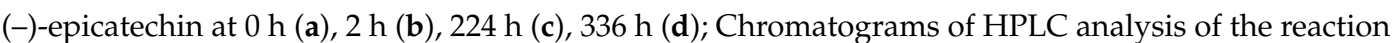
between malvidin-3-glucoside and (-)-epicatechin at 0h (e), $2 \mathrm{~h} \mathrm{(f),} 224 \mathrm{~h} \mathrm{(g),} 336 \mathrm{~h} \mathrm{(h);} \mathrm{Chromatograms}$ of HPLC analysis of the reaction between peonidin-3-glucoside and (-)-epicatechin at $0 \mathrm{~h}(\mathbf{i}), 2 \mathrm{~h}(\mathbf{j})$, $224 \mathrm{~h}(\mathbf{k}), 336 \mathrm{~h}(\mathbf{1})$.

Table 1. Rate constant of reaction between anthocyanin and (-)-epicatechin mediated by acetaldehyde at different reaction conditions in model wine solution.

\begin{tabular}{|c|c|c|c|c|}
\hline \multirow{2}{*}{$\begin{array}{c}\text { Reactants } \\
\text { (anthocyanin + EC) }\end{array}$} & \multicolumn{4}{|c|}{$\begin{array}{c}\text { Reaction Condition } \\
\text { (Temperature, Molar Ratio (anthocyanin/EC/acetaldehyde)) }\end{array}$} \\
\hline & $\mathrm{pH}=1.7$ & Rate Constant $K\left(\mathrm{~h}^{-1}\right)$ & $\mathrm{pH}=3.2$ & Rate Constant $K\left(\mathrm{~h}^{-1}\right)$ \\
\hline \multirow{3}{*}{$\mathrm{Mv}+\mathrm{EC}$} & $25^{\circ} \mathrm{C}, 1: 3: 10$ & 0.0565 & $25^{\circ} \mathrm{C}, 1: 3: 10$ & 0.0065 \\
\hline & $25^{\circ} \mathrm{C}, 1: 6: 10$ & 0.0919 & $25^{\circ} \mathrm{C}, 1: 6: 10$ & 0.0090 \\
\hline & $30^{\circ} \mathrm{C}, 1: 6: 10$ & 0.1763 & $30{ }^{\circ} \mathrm{C}, 1: 6: 10$ & 0.0191 \\
\hline $\mathrm{Cy}+\mathrm{EC}$ & $25^{\circ} \mathrm{C}, 1: 6: 10$ & 0.1005 & $25^{\circ} \mathrm{C}, 1: 6: 10$ & 0.0066 \\
\hline$P n+E C$ & $25^{\circ} \mathrm{C}, 1: 6: 10$ & 0.1630 & $25^{\circ} \mathrm{C}, 1: 6: 10$ & 0.0101 \\
\hline
\end{tabular}

Abbreviations: Cy, cyanidin-3-glucoside; EC, (-)-epicatechin; Mv, malvidin-3-glucoside; Pn, peonidin-3-glucoside.

\subsection{Structural Identification}

The structures of ethyl-linked anthocyanin-flavanol pigments were identified based on molecular ions and MS $^{2}$ fragmentation using HPLC-QTOF-MS and ECD analysis.

Representative TIC chromatograms of the reaction mixture are shown in Figure 2. The major ions observed in the MS/MS ${ }^{2}$ spectra are presented in Table 2. In the three reaction mixtures, peaks 3 and 
4 (Figure 2) are suggested as the two isomers, showing ions at $m / z 809.2293$ and $809.2289,765.2029$ and 765.2025, and 779.2184 and 779.2182, corresponding to the products from condensation reactions between malvidin-3-glucoside, cyanidin-3-glucoside, and peonidin-3-glucoside and (-)-epicatechin mediated by acetaldehyde, respectively. These molecular masses and fragmentation ions were matched well with previously studies [46-48]. The C-8 position of the A-ring of either flavanols or anthocyanins was identified as the preferred substitution site for such indirect condensation reactions [30]. Due to the presence of an asymmetric carbon in the ethyl bridge, the two isomers exhibited the same fragmentation patterns. Peak 3 of each reaction mixture was studied as being representative of its fragmentation pattern. Malvidin-3-glucoside-ethyl-epicatechin (1) had an ion with $\mathrm{m} / z$ 809.2293. The ion with $\mathrm{m} / z 519.1502$ could be derived from the loss of one (-)-epicatechin (290 Da). The ion with $\mathrm{m} / z 647.1760$ could result from the loss of one glucose moiety (162 Da) and the ion with $m / z 357.0974$ might be formed from the further loss of one (-)-epicatechin (290 Da) (Figure 3A). This fragmentation pattern was consistent with that which has been reported in literature [46,49]. Cyanidin-3-glucoside-ethyl-epicatechin (1) had an ion with $m / z$ 765.2029. The ion with $m / z 603.1496$ could be derived from the loss of one glucose moiety (162 Da). The ion with $m / z 475.1239$ could result from the loss of one (-)-epicatechin (290 Da), and the ion with $\mathrm{m} / z 313.0713$ might be formed from the further loss of one glucose moiety (162 Da) (Figure 3B). Peonidin-3-glucoside-ethyl-epicatechin (1) had ions with $m / z$ of $779.2184,617.1655,489.1395$, and 327.0872, indicating a similar fragmentation pattern to that of cyanidin-3-glucoside-ethyl-epicatechin (1), as shown in Figure 3C. These results were in agreement with previous studies $[47,50,51]$. Molecular ions combined with fragmentation patterns confirmed the identification of the six ethyl-linked anthocyanin-flavanol pigments through comparison with literatures [47-51]. In addition, ions with $m / z$ of 1125.3240, 1081.2977, and 1095.3126 were detected from three reaction mixtures containing malvidin-3-glucoside, cyanidin-3-glucoside, and peonidin-3-glucoside as the reactant, respectively. It is speculated that these correspond to the structure of one anthocyanin molecule and two (-)-epicatechin molecules linked by two molecule ethyl bridge. However, the ion abundance was weak and their content was too low for further study or separation.

Table 2. Anthocyanins and condensation reaction products detected by ESI-MS ${ }^{2}$.

\begin{tabular}{ccccc}
\hline Sample & No. & Compound & [M] $^{+}$ & MS $^{\mathbf{2}}$ Product Ions $(\boldsymbol{m} / \mathbf{z})$ \\
\hline \multirow{3}{*}{ Mv + EC } & 1 & Mv & 493.1344 & 331.0812 \\
& 2 & Mv-ethyl-EC (1) & 809.2293 & $647.1760,519.1502,357.0974$ \\
& 3 & Mv-ethyl-EC (2) & 809.2289 & $647.1763,519.1497,357.0976$ \\
& 4 & Mv-ethyl-EC-ethyl-EC & 1125.3240 & \\
\hline \multirow{4}{*}{ Cy + EC } & 1 & Cy & 449.1082 & 287.0551 \\
& 2 & Cy-ethyl-EC (1) & 765.2029 & $603.1496,475.1239,313.0713$ \\
& 3 & Cy-ethyl-EC (2) & 765.2025 & $603.1500,475.1235,313.0715$ \\
Pn + EC & 4 & Cy-ethyl-EC-ethyl-EC & 1081.2977 & \\
& 1 & Pn & 463.1239 & 301.0707 \\
& 2 & Pn-ethyl-EC (1) & 779.2184 & $617.1655,489.1395,327.0872$ \\
& 3 & Pn-ethyl-EC (2) & 779.2182 & $617.1659,489.1391,327.0872$ \\
\hline
\end{tabular}

Abbreviations: Cy, cyanidin-3-glucoside; EC, (-)-epicatechin; Mv, malvidin-3-glucoside; Pn, peonidin-3-glucoside. 


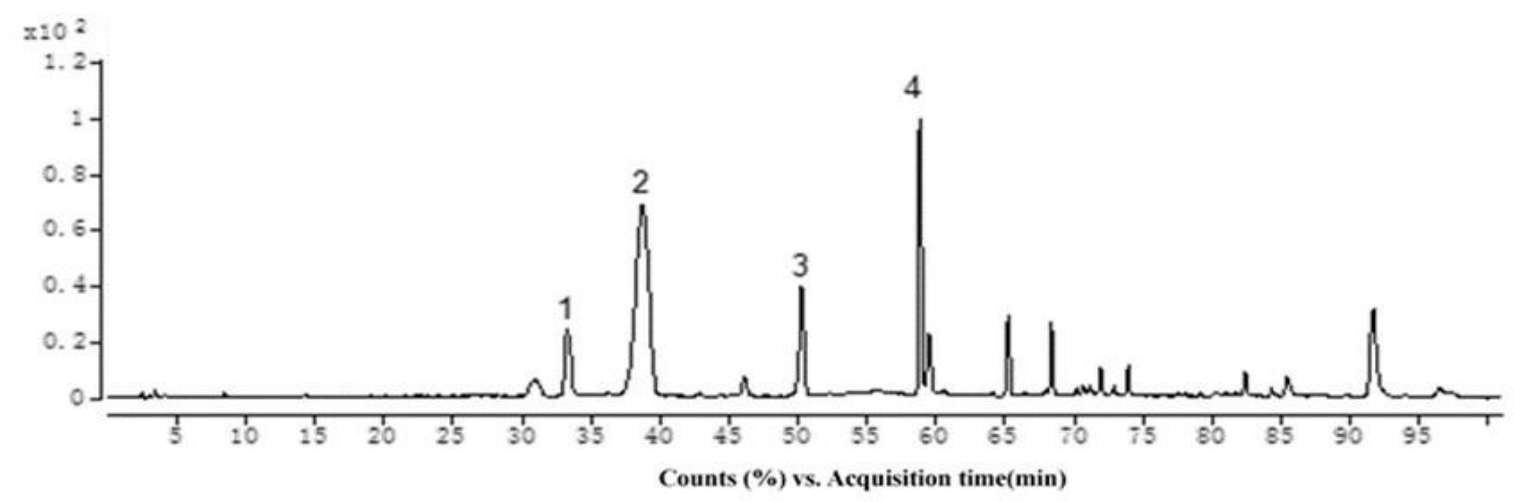

(A)

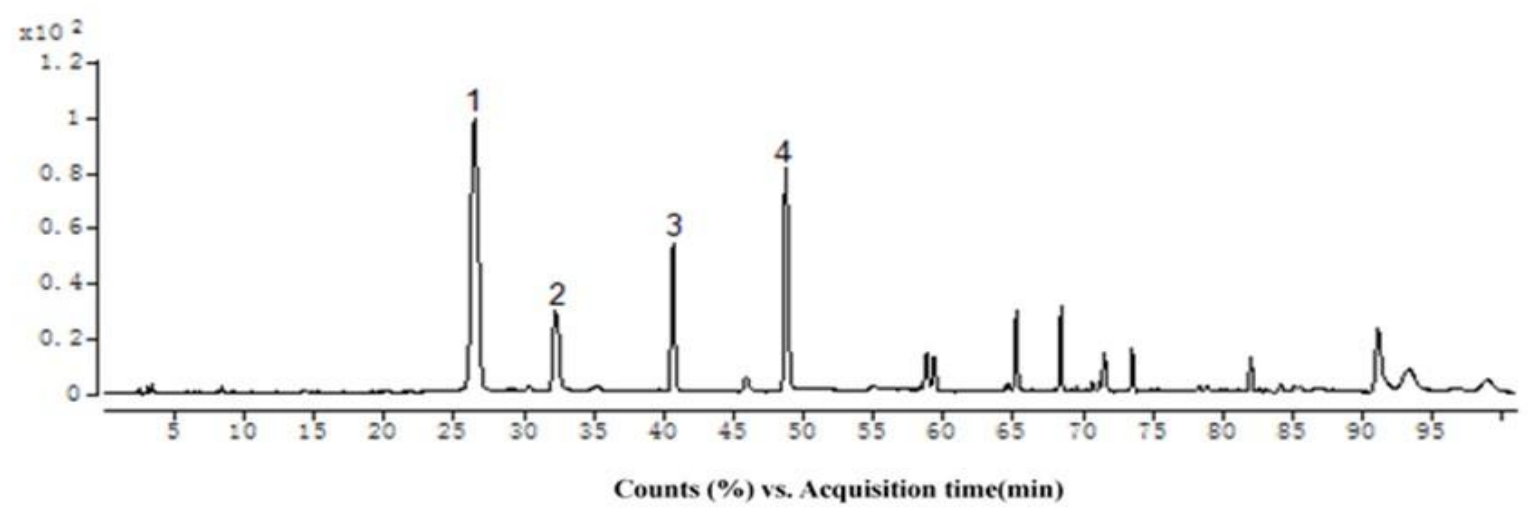

(B)

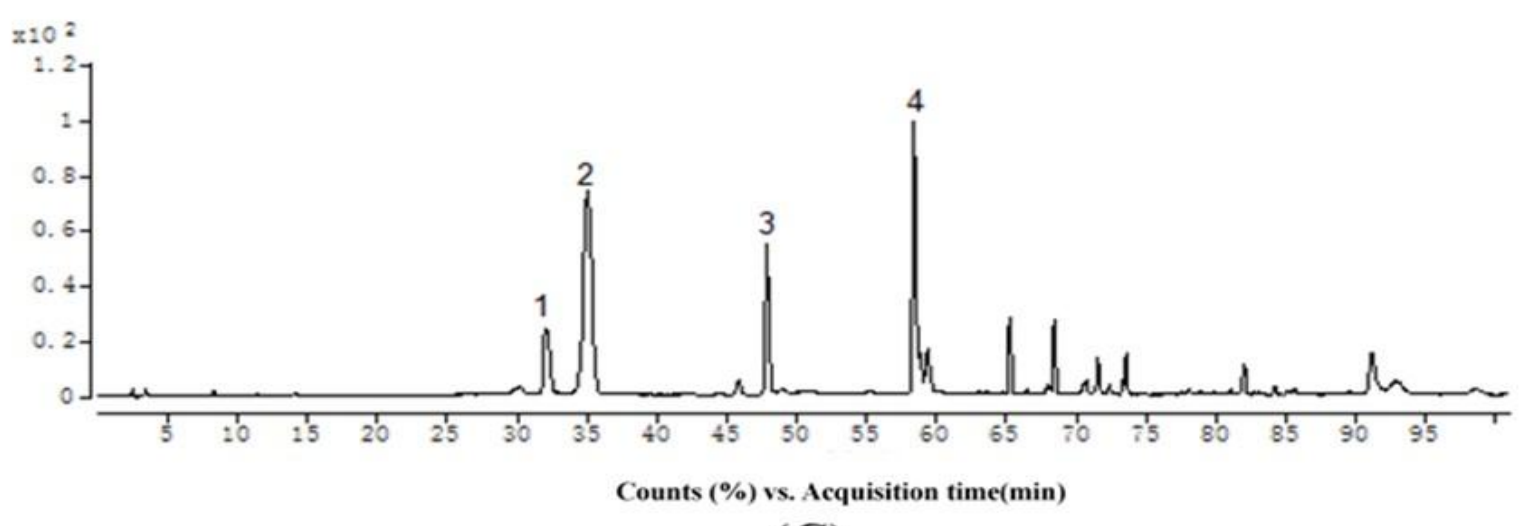

(C)

Figure 2. (A) Representative TIC chromatogram in positive mode of malvidin-3-glucoside and condensation products: 1. (-)-epicatechin, 2. malvidin-3-glucoside, 3. malvidin-3-glucoside-ethylepicatechin (1), 4. malvidin-3-glucoside-ethyl-epicatechin (2); (B) Representative TIC chromatogram in positive mode of cyanidin-3-glucoside and condensation products: 1 . cyanidin-3-glucoside, 2. (-)-epicatechin, 3. cyanidin-3-glucoside-ethyl-epicatechin (1), 4. cyanidin-3-glucoside-ethyl-epicatechin (2); (C) Representative TIC chromatogram in positive mode of peonidin-3-glucoside and condensation products: 1. (-)-epicatechin, 2. peonidin-3-glucoside, 3. peonidin-3-glucoside-ethyl-epicatechin (1), 4. peonidin-3-glucoside-ethyl-epicatechin (2). 

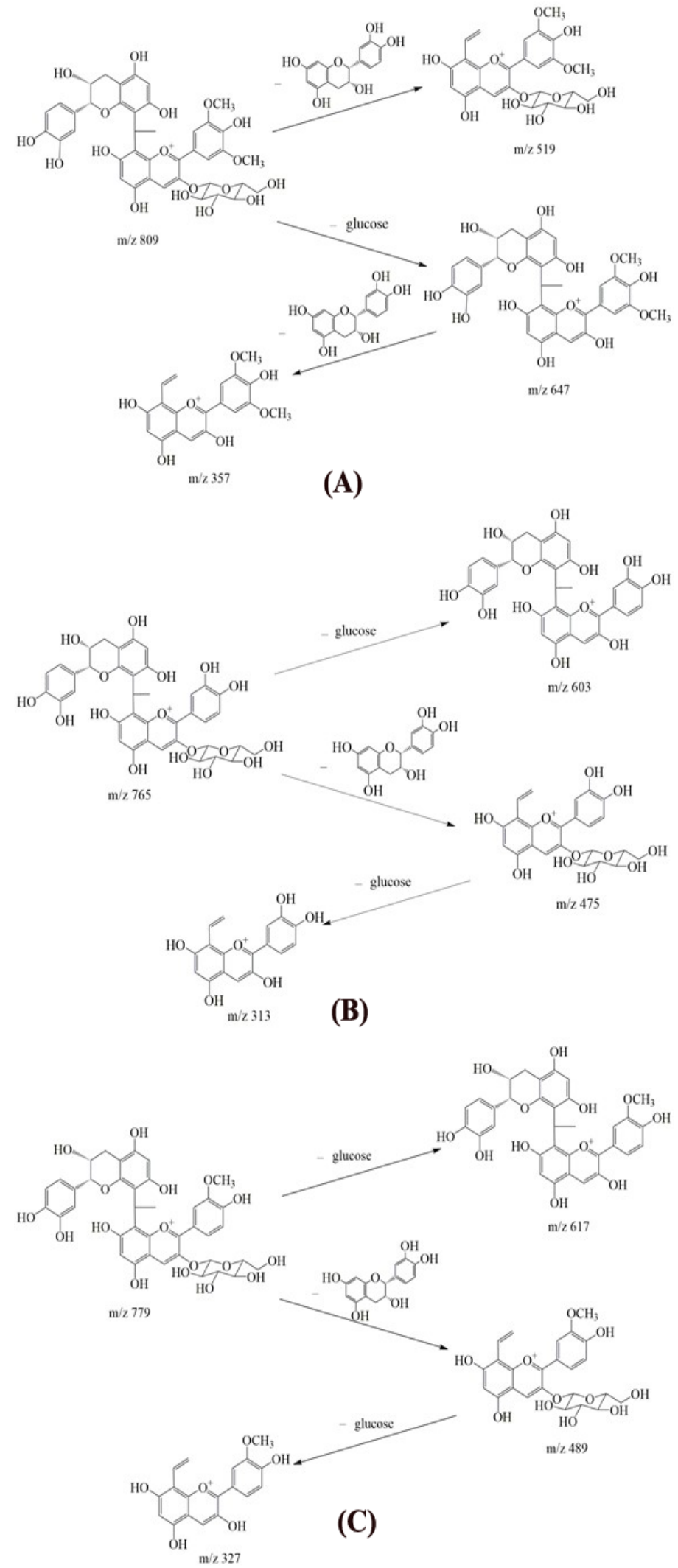

Figure 3. The main fragmentation pathways of condensation products of malvidin-3-glucoside-ethylepicatechin (1) (A); cyanidin-3-glucoside-ethyl-epicatechin (1) (B) and peonidin-3-glucoside-ethylepicatechin (1) (C). 
ECD analysis was used to determine the absolute configurations and conformations of chiral molecules. This technique has been widely used for the structural elucidation of natural products. The determination procedure was based on comparing the calculated and experimental ECD spectra [52]. The six ethyl-linked anthocyanin-flavanol pigment molecules contained a chiral carbon atom at the same position, thus ECD calculation was applied to cyanidin-3-glucoside-ethyl-epicatechin (1), and the configurations of the other five molecules were determined by comparison with it. The ECD spectra of cyanidin-3-glucoside-ethyl-epicatechin (1) containing both $R$ configuration and $S$ configuration were predicted using SpecDis software, and the weighted average method was used to obtain the spectra. The predicted spectra were compared to the experimental spectrum (Figure 4). The results indicate that the ECD spectrum of $S$ configuration is consistent with the experimental spectrum, whereas that of the $R$ configuration shows obvious differences from the experimental spectrum at 230-250 nm. Therefore, the absolute configuration of cyanidin-3-glucoside-ethyl-epicatechin (1) was identified as $S$ configuration. Compared with the experimental spectrum (Figure 5): cyanidin-3-glucoside-ethyl-epicatechin (1), malvidin-3-glucoside-ethyl-epicatechin (1), and peonidin-3-glucoside-ethyl-epicatechin (1) were all identified as $S$ configuration, whereas cyanidin-3-glucoside-ethyl-epicatechin (2), malvidin-3-glucoside-ethyl-epicatechin (2), and peonidin-3-glucoside-ethyl-epicatechin (2) were identified as $R$ configuration.

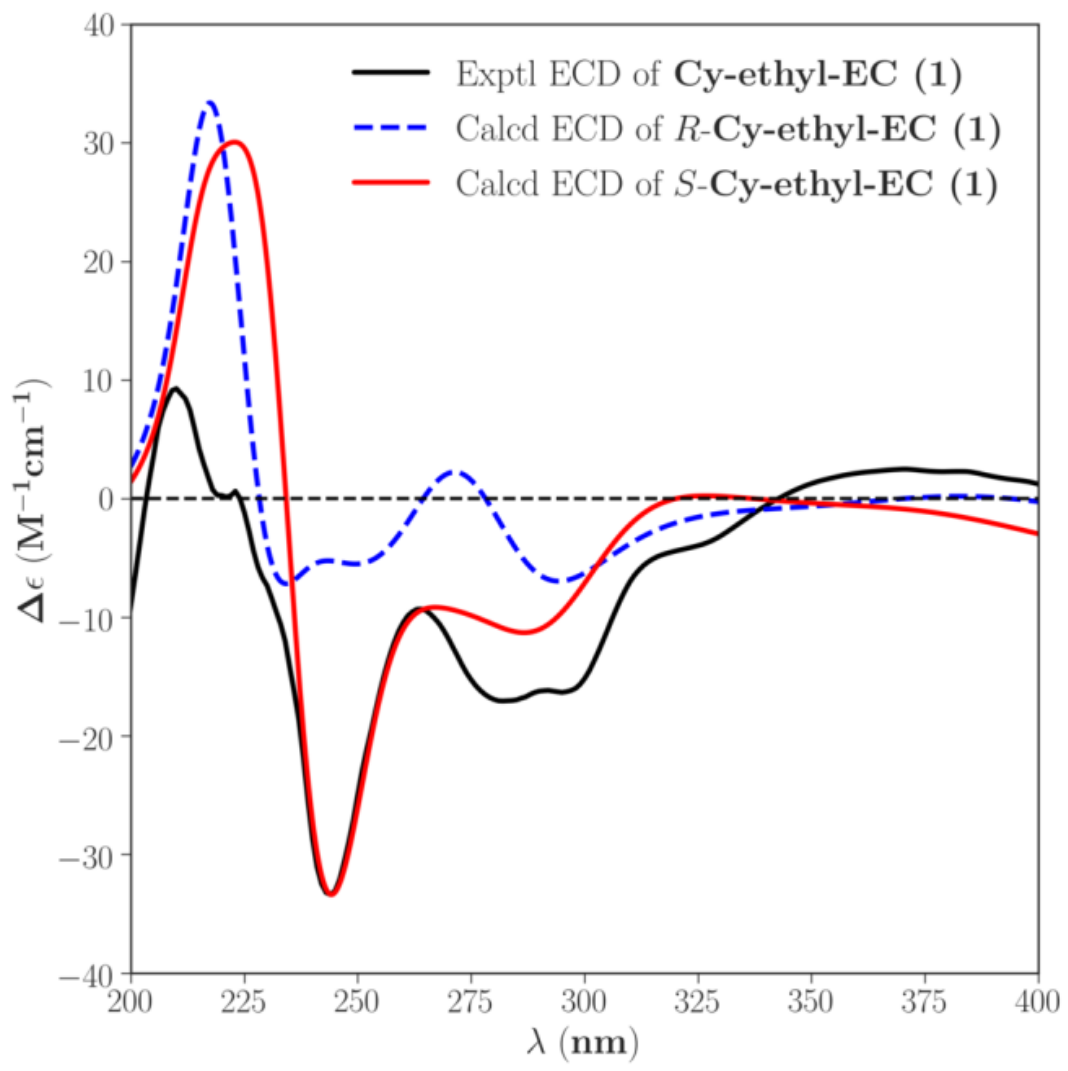

Figure 4. Calculated ECD spectra of configurations $R$ and $S$ were compared with the experimental. 
A

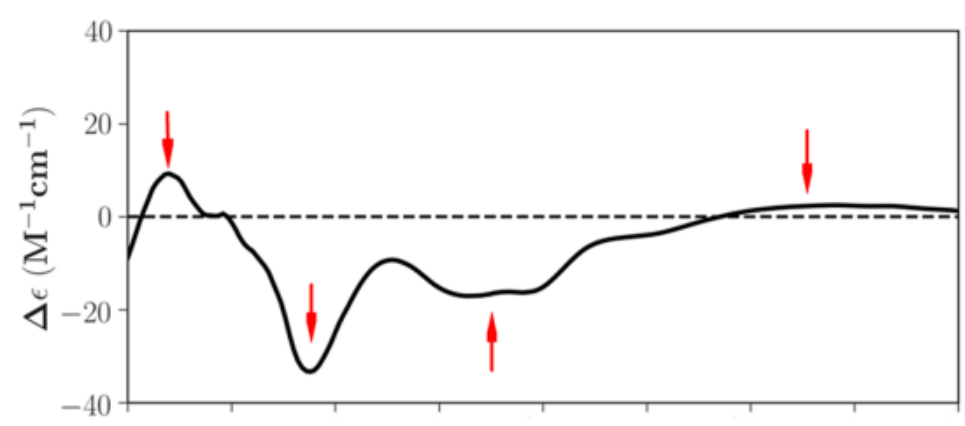

B

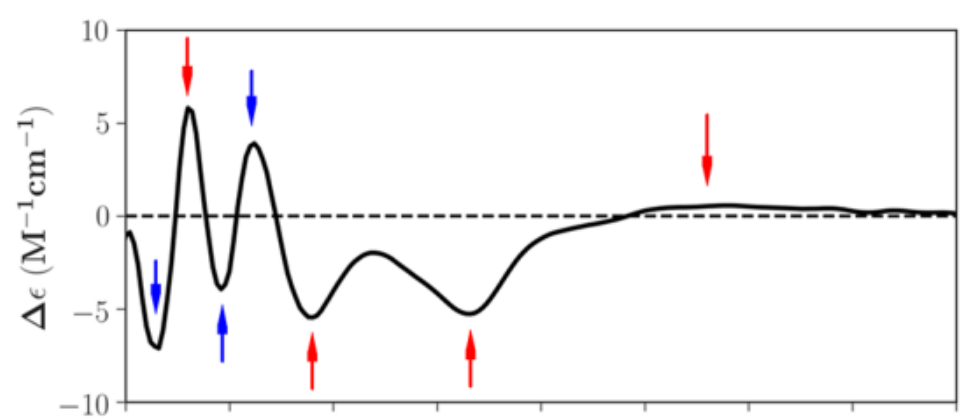

C

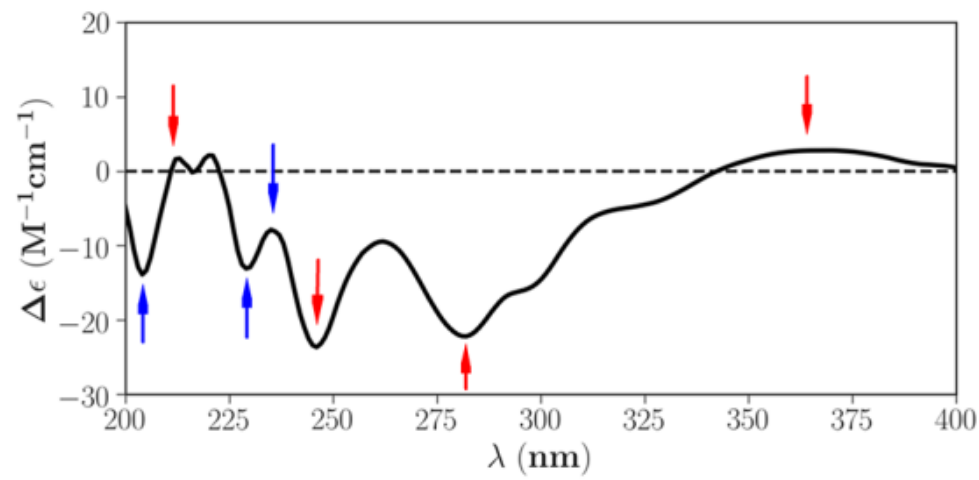

Figure 5. The comparison of CD spectrum of cyanidin-3-glucoside-ethyl-epicatechin (1) (A), malvidin-3-glucoside-ethyl-epicatechin (1) (B) and peonidin-3-glucoside-ethyl-epicatechin (1) (C).

\subsection{Isolation of Individual Ethyl-Linked Anthocyanin-Flavanol Pigments by Preparative HPLC}

Six ethyl-linked anthocyanin-flavanol pigments were isolated and further purified from the reaction solution when the maximum product yields were reached using preparative HPLC under the optimized conditions (Figure 6). Yields of $3.3 \mathrm{mg}$ malvidin-3-glucoside-ethyl-epicatechin (S), $4.1 \mathrm{mg}$ malvidin-3-glucoside-ethyl-epicatechin $(R), 2.2 \mathrm{mg}$ cyanidin-3-glucoside-ethyl-epicatechin $(S), 6.5 \mathrm{mg}$ cyanidin-3-glucoside-ethyl-epicatechin $(R), 3.3 \mathrm{mg}$ peonidin-3-glucoside-ethyl-epicatechin $(S)$, and $5.1 \mathrm{mg}$ peonidin-3-glucoside-ethyl-epicatechin $(R)$ were obtained from conversion of $20 \mathrm{mg}$ malvidin-3-glucoside, cyanidin-3-glucoside, and peonidin-3-glucoside, respectively. The purities of all the products exceeded $95 \%$. The possible impurities in each compound were composed of its isomers or trace amounts of other pigments [44] that had similar properties with it. Therefore, sufficient quantities of individual, high-purity ethyl-linked anthocyanin-flavanol pigments were obtained to provide material guarantee for the study of antioxidant activity. 

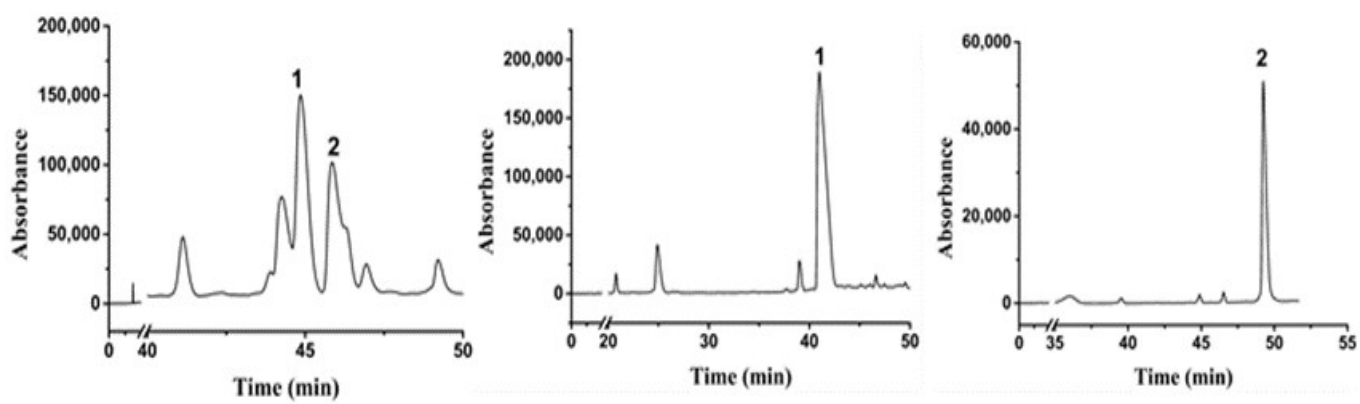

(A)
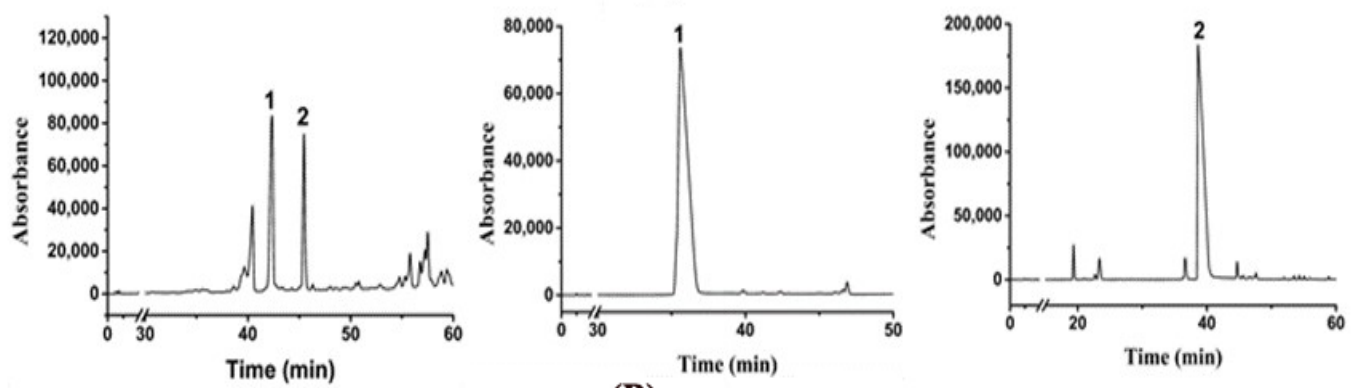

(B)
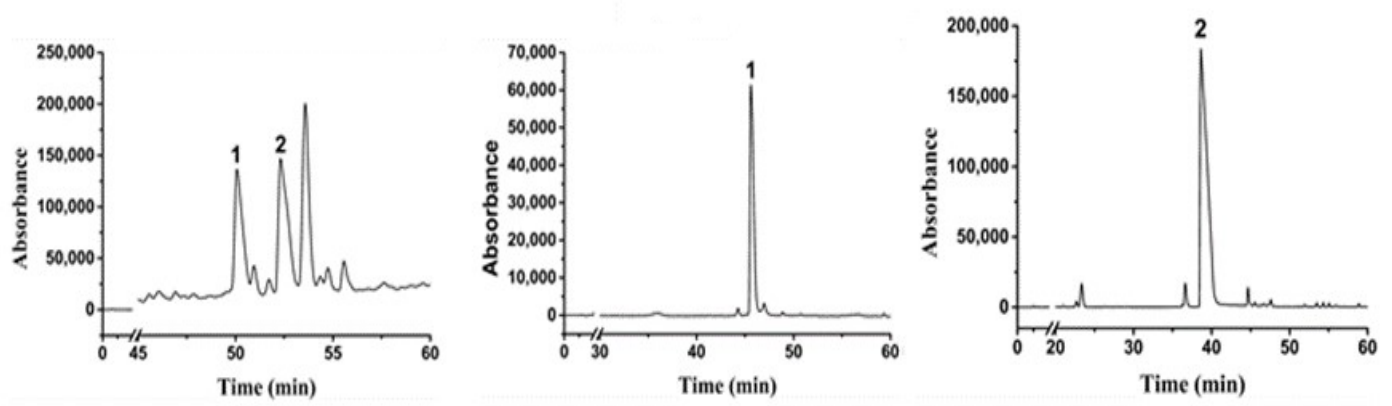

(C)

Figure 6. Preparative-HPLC chromatograms of six ethyl-linked anthocyanin-flavanol pigments. (A) 1: malvidin-3-glucoside-ethyl-epicatechin $(S)$; 2: malvidin-3-glucoside-ethyl-epicatechin $(R)$. (B) 1: cyanidin-3-glucoside-ethyl-epicatechin $(S)$; 2: cyanidin-3-glucoside-ethyl-epicatechin $(R)$. (C) 1: peonidin-3-glucoside-ethyl-epicatechin $(S)$; 2: peonidin-3-glucoside-ethyl-epicatechin $(R)$.

\subsection{Antioxidant Activity}

Many studies have shown that flavanols and anthocyanins possess strong antioxidant activities in vitro and in vivo [7,53-56]. However, there are presently few studies on the antioxidant activities of ethyl-linked anthocyanin-flavanol pigments. For determination of six individual ethyl-linked anthocyanin-flavanol pigments as well as their precursor anthocyanins and (-)-epicatechin, three different assay methods (i.e., DPPH, ABTS, and FRAP) were used and the results are presented in Table 3. 
Table 3. Antioxidant activities and linear ranges of (-)-epicatechin, anthocyanins, ethyl-linked anthocyanin-flavanol pigments and antioxidants by DPPH, ABTS, and FRAP assays.

\begin{tabular}{|c|c|c|c|c|c|c|}
\hline \multirow{2}{*}{ Compounds } & \multicolumn{2}{|c|}{$\mathrm{DPPH}(\mu \mathrm{mol} / \mathrm{L})$} & \multicolumn{2}{|c|}{ ABTS $(\mu \mathrm{mol} / \mathrm{L})$} & \multicolumn{2}{|c|}{ FRAP $(\mu \mathrm{mol} / \mathrm{L})$} \\
\hline & EC50 & Linear Range & EC50 & Linear Range & FRAP Value & Linear Range \\
\hline $\mathrm{Pn}$ & $189 \pm 3^{c}$ & $24.8-198.5$ & $107 \pm 2^{c}$ & $19.8-148.8$ & $10.3 \pm 0.0059 \mathrm{~g}$ & $24.3-194.8$ \\
\hline $\mathrm{Mv}$ & $190 \pm 10^{c}$ & $24.3-194.8$ & $106 \pm 2^{c}$ & $19.5-146.1$ & $8.9 \pm 0.0070^{h}$ & $24.8-248.1$ \\
\hline $\mathrm{Cy}$ & $170 \pm 5^{\mathrm{d}}$ & $48.9-244.6$ & $103 \pm 1^{d}$ & $19.6-146.7$ & $10.8 \pm 0.0056^{\mathrm{f}}$ & $12.2-146.7$ \\
\hline EC & $153 \pm 4^{\mathrm{e}}$ & $50.2-301.2$ & $99 \pm 1^{\mathrm{e}}$ & $50.2-200.8$ & $11.5 \pm 0.0069^{\mathrm{e}}$ & $25.1-200.8$ \\
\hline Pn-ethyl-EC (S) & $130 \pm 4^{\mathrm{f}}$ & 24-192 & $95 \pm 2^{\mathrm{f}}$ & 24-192 & $12.2 \pm 0.0045^{\mathrm{d}}$ & 24-192 \\
\hline Pn-ethyl-EC (R) & $121 \pm 2^{f}$ & 24.5-196.1 & $90 \pm 2^{g}$ & $24.5-196.1$ & $12.4 \pm 0.0079^{d}$ & $25.6-205$ \\
\hline Mv-ethyl-EC (S) & $132 \pm 1^{\mathrm{f}}$ & $25.6-205$ & $86 \pm 1^{h}$ & $25.6-153.8$ & $13.3 \pm 0.0069^{b}$ & $24.8-198$ \\
\hline Mv-ethyl-EC $(R)$ & $135 \pm 2^{f}$ & $24.8-198$ & $87 \pm 2^{g, h}$ & $29.7-118.8$ & $12.9 \pm 0.0074^{\mathrm{c}}$ & $12.2-145.8$ \\
\hline Cy-ethyl-EC (S) & $83 \pm 2 g$ & $12.2-97.2$ & $79 \pm 3^{i}$ & $24.3-116.7$ & $12.4 \pm 0.0061^{\mathrm{d}}$ & $12.2-146$ \\
\hline Cy-ethyl-EC $(R)$ & $80 \pm 3^{g}$ & $12.2-97.3$ & $77 \pm 3^{i}$ & $24.3-116.8$ & $16.5 \pm 0.0048^{a}$ & $12.5-150$ \\
\hline $\mathrm{V}_{\mathrm{C}}$ & $1030 \pm 27^{a}$ & $125.5-2007.6$ & $469 \pm 4^{\mathrm{a}}$ & $200.8-702.6$ & & \\
\hline Trolox & $921 \pm 17^{b}$ & 200.4-1002 & $447 \pm 2^{b}$ & 200.4-701.4 & $2.2 \pm 0.0049^{\mathrm{i}}$ & 200.4-1002 \\
\hline $\mathrm{FeSO}_{4}$ & & & & & & 400-2000.1 \\
\hline
\end{tabular}

Abbreviations: Cy, cyanidin-3-glucoside; EC, (-)-epicatechin; Mv, malvidin-3-glucoside; Pn, peonidin-3-glucoside. EC50 values or FRAP values were presented as mean \pm standard deviation of three independent experiments $(n=3)$. Superscript $(\mathrm{a}-\mathrm{i})$ in a column mean significant differences, $p<0.05$.

The scavenging capacities of ethyl-linked anthocyanin-flavanol pigments, anthocyanins, and (-)-epicatechin on DPPH. were expressed as EC50 values with two common antioxidants (Vc and Trolox) as controls. The EC50 values of the three anthocyanins, (-)-epicatechin, and six ethyl-linked anthocyanin-flavanol pigments were between $80 \mu \mathrm{mol} / \mathrm{L}$ and $190 \mu \mathrm{mol} / \mathrm{L}$, much less than the 921 and $1030 \mu \mathrm{mol} / \mathrm{L}$ of Trolox and Vc. Thus, anthocyanins, (-)-epicatechin, and the six ethyl-linked anthocyanin-flavanol pigments all exhibited stronger activities for radical scavenging than $V_{c}$ and Trolox. The antioxidant abilities were found to decrease in this sequence: ethyl-linked anthocyanin-flavanol pigments $>(-)$-epicatechin $>$ anthocyanins. Cyanidin-3-glucoside showed the highest antioxidant activity among the three anthocyanins, followed by malvidin-3-glucoside and peonidin-3-glucoside with no significant difference. Comparison of EC50 values among the six ethyl-linked anthocyanin-flavanol pigments demonstrated that cyanidin-3-glucoside-ethyl-epicatechin which was polymerized from cyanidin-3-glucoside possessed better antioxidant capacities, and that there was no significant difference between the isomers of the ethyl-linked anthocyanin-flavanol pigments.

ABTS assays showed similar results to DPPH assays. Anthocyanins, (-)-epicatechin, and six ethyl-linked anthocyanin-flavanol pigments showed stronger ABTS. ${ }^{+}$scavenging activities compared with Vc and Trolox. Ethyl-linked anthocyanin-flavanol pigments exhibited more powerful ABTS. ${ }^{+}$scavenging activity than their precursor anthocyanins and (-)-epicatechin, and cyanidin-3-glucoside-ethyl-epicatechin $(R)$ showed the most powerful antioxidant capacity among them. In comparing anthocyanins, cyanidin-3-glucoside exhibited the strongest scavenging capacity, and no significant difference was seen in antioxidant activity between peonidin-3-glucoside and malvidin-3-glucoside. Ethyl-linked anthocyanin-flavanol pigment isomers showed similar antioxidant activities.

The reducing abilities on $\mathrm{Fe}^{3+}$-TPTZ (FRAP values) of ethyl-linked anthocyanin-flavanol pigments, anthocyanins, and (-)-epicatechin were calculated by substituting the absorbance values of the analytes into the regression equation of $\mathrm{FeSO}_{4}$. The results indicated that anthocyanins, (-)-epicatechin, and ethyl-linked anthocyanin-flavanol pigments are all powerful ferric-reducing antioxidants, compared with the well-known antioxidant Trolox. The ferric ion-reducing activities were found to follow this sequence: ethyl-linked anthocyanin-flavanol pigments $>(-)$-epicatechin $>$ anthocyanins. Among all the analytes, cyanidin-3-glucoside-ethyl-epicatechin $(R)$ showed the highest antioxidant activity and malvidin-3-glucoside the lowest.

The comprehensive results of the three assays demonstrate that anthocyanins, (-)-epicatechin, and ethyl-linked anthocyanin-flavanol pigments all possess strong antioxidant activity. (-)-Epicatechin showed a slightly greater antioxidant capacity than the individual anthocyanins, which was consistent with the results of our previous study [57]. Among the three anthocyanins, cyanidin-3-glucoside 
presented the highest antioxidant activity followed by peonidin-3-glucoside and malvidin-3-glucoside, in agreement with published studies [58,59]. Previous studies indicated that some pyranoanthocyanins have a higher antioxidant potential than their precursor anthocyanins, whereas others do not $[60,61]$. In this study, the antioxidant activities of ethyl-linked anthocyanin-flavanol pigments were significantly higher than their precursor anthocyanins, and cyanidin-3-glucoside-ethyl-epicatechin possessed the highest antioxidant activity. In addition, the chiral structure of the ethyl-linked anthocyanin-flavanol pigments showed similar antioxidant capacities. Antioxidant activity was related to the presence of phenolic hydroxyl group. Theoretically, more phenolic hydroxyl groups would be associated with stronger antioxidant activity. The number of phenolic hydroxyl groups in one molecule of ethyl-linked anthocyanin-flavanol pigment was the sum of anthocyanin and (-)-epicatechin, thus ethyl-linked anthocyanin-flavanol pigments showed higher antioxidant activity, which was verified in practice by DPPH, ABTS, and FRAP assays. In addition, hydroxylation and/or methoxylation and their position on the B ring might have an influence on antioxidant capacity [62,63]. Antioxidant activity might be enhanced as a result of the hydroxyl at the $\mathrm{C}-3^{\prime}$ position on the $\mathrm{B}$ ring or subdued due to methylation at the $C-3^{\prime}$ and / or $C-5^{\prime}$ position [64]. This might be the cause of the differing antioxidant activities of the anthocyanin unit. This is the first reported experimental determination and evaluation of the antioxidant activities of these six ethyl-linked anthocyanin-flavanol pigments, and the findings indicate markedly enhanced antioxidant activity when anthocyanins formed into ethyl-linked anthocyanin-flavanol pigments. This study provides an experimental foundation and theoretical basis for the development and application of ethyl-linked anthocyanin-flavanol pigments as antioxidants.

The correlations of antioxidant activities, determined by ABTS, DPPH, and FRAP assays, were estimated using Pearson's correlation coefficient (two-tailed). High correlations were found among the assays. The strongest correlation was observed between DPPH and ABTS assays $(r=0.991, P<0.001)$, and the weakest between FRAP and ABTS assays $(r=-0.882, P<0.001)$. The DPPH assay showed a strong positive correlation with the ABTS assay, while the FRAP assay was highly negatively correlated with DPPH and ABTS assays, with FRAP and DPPH assays showing the strongest negative correlation $(r=-0.901, P<0.001)$. Therefore, the results obtained using the three methods are reliable.

\section{Materials and Methods}

\subsection{Chemicals and Materials}

Acetaldehyde was purchased from Aladdin (Shanghai, China). L-ascorbic acid $\left(\mathrm{V}_{\mathrm{C}}\right)$ was purchased from Fluka (Buchs, Switzerland). 2,2-Diphenyl-1-picrylhydrazyl (DPPH), 2,2'-Azino-bis (3-ethylbenzothiazoline-6-sulfonic acid) diammonium salt (ABTS), 2,4,6-Tris(2-pyridyl)-s-triazine (TPTZ), ( \pm )-6-Hydroxy-2,5,7,8-tetramethylchromane-2-carboxylic acid (Trolox) were purchased from Sigma-Aldrich (St. Louis, MO, USA). Blueberry extract was purchased from Tianjin Jianfeng Natural Product R\&D Co., Ltd. (Tianjin, China). Red wine extract was provided by Polyphenol Laboratory of Pólo Dois Portos/INIAV (Lisbon, Portugal).

All organic solvents used for HSCCC (analytical grade) and HPLC (chromatographic grade) were purchased from Chemical Branch of Shandong Yuwang Industrial Co., Ltd. (Shandong, China).

\subsection{Preparation of Anthocyanins and Flavanols by HSCCC}

Malvidin-3-glucoside and peonidin-3-glucoside were isolated from red wine extract by HSCCC (TBE 300B, Tauto Biotechnique Company, Shanghai, China) and combined with preparative HPLC (Waters e2695, Waters, Milford, MA, USA), as described previously in our laboratory [57]. To isolate enough anthocyanins for condensation reactions, red wine extract fermented for 7 days, which is rich in individual anthocyanins, was used. The optimized HSCCC condition was a solvent system consisting of the two phases of methyl tert-butyl ether- $n$-butanol-acetonitrile-water (1-40-1-50, acidified with $0.01 \%$ trifluoroacetic acid, $v / v$ ) with a flow rate of $2 \mathrm{~mL} / \mathrm{min}$. One hundred milligrams of red wine 
extract dissolved in $20 \mathrm{~mL}$ lower phase were injected into the apparatus. The HSCCC rotary speed was set at $950 \mathrm{rpm}$ and the detection wavelength was $525 \mathrm{~nm}$. The fractions were collected manually and evaporated to remove organic solvents. The further purification of malvidin-3-glucoside and peonidin-3-glucoside was performed using preparative HPLC, with the mobile phase consisting of $0.2 \%$ formic acid-water (solvent $\mathrm{A}$ ) and $0.2 \%$ formic acid-acetonitrile (solvent $\mathrm{B}$ ). The elution conditions were as follows: malvidin-3-glucoside $(12 \% \mathrm{~B}, 4 \mathrm{~mL} / \mathrm{min})$ and peonidin-3-glucoside $(10 \% \mathrm{~B}, 3.5 \mathrm{~mL} / \mathrm{min})$. The temperature of the column was set at $30{ }^{\circ} \mathrm{C}$.

Blueberry is a rich source of anthocyanins, especially cyanidin-3-glucoside. Thus, cyanidin-3-glucoside was isolated from blueberry extract using HSCCC. A solvent system composed of methyl tert-butyl ether-n-butanol-acetonitrile-water (1-40-1-50, acidified with 0.01\% trifluoroacetic acid, $v / v$ ) with a flow rate of $2 \mathrm{~mL} / \mathrm{min}$ was used as the optimized HSCCC condition. The $20 \mathrm{~mL}$ lower phase with $100 \mathrm{mg}$ of blueberry extract was injected through the sample loop. The apparatus was run at $950 \mathrm{rpm}$ and the UV detector was set at $525 \mathrm{~nm}$. The preparative HPLC was used to purify high-purity cyanidin-3-glucoside and the elution condition was $12 \%$ B (A: $0.2 \%$ formic acid-water; B: acetonitrile) with a flow rate of $4 \mathrm{~mL} / \mathrm{min}$ and a column temperature of $30^{\circ} \mathrm{C}$.

Typically, procyanidins in cacao beans are mainly composed of (-)-epicatechin structural units. To obtain (-)-epicatechin on a large scale as a reactant for condensation reactions, (-)-epicatechin was separated from cacao-bean phenolic extract using HSCCC based on our previous work [65]. The cacao-bean phenolic extract was prepared according to our previously published procedure [66]. The optimized HSCCC condition was as follows: The two-phase solvent system was $n$-hexane-ethyl acetate-water $(1: 50: 50, v / v)$ and flow rate was $3 \mathrm{~mL} / \mathrm{min}$. The sample solution was prepared by dissolving $300 \mathrm{mg}$ of the cacao-bean phenolic extract in the $20 \mathrm{~mL}$ lower phase. Both the tail-head and head-tail elution modes were used with a rotary speed of $950 \mathrm{rpm}$ and a detection wavelength of $280 \mathrm{~nm}$. (-)-Epicatechin was further purified through preparative HPLC in large scale with water (solvent A) and methanol (solvent B) as the mobile phase with an elution condition of $30 \%$ B. The column temperature was set at $30^{\circ} \mathrm{C}$. The flow rate of the mobile phase was fixed at $3 \mathrm{~mL} / \mathrm{min}$.

\subsection{Model Wine Solution}

The model wine solution used for the condensation reactions was composed of $12 \%$ ethanol and $5 \mathrm{~g} / \mathrm{L} \mathrm{1-tartaric} \mathrm{acid} \mathrm{dissolved} \mathrm{in} \mathrm{water,} \mathrm{adjusted} \mathrm{to} \mathrm{pH} 3.2$ with $1 \mathrm{~mol} / \mathrm{L} \mathrm{HCl}$.

\subsection{Optimization of the Condensation Reaction between Anthocyanins and Flavanols Mediated by Acetaldehyde}

Two $\mathrm{pH}$ values, 3.2 and 1.7, were chosen for the model solutions and the $\mathrm{pH}$ was adjusted by the addition of $1 \mathrm{~mol} / \mathrm{L} \mathrm{HCl}$ or $1 \mathrm{~mol} / \mathrm{L} \mathrm{NaOH}$. The $\mathrm{pH}$ values of 3.2 and 1.7 correspond to the $\mathrm{pH}$ of red wine and the $\mathrm{pH}$ at which anthocyanins are mainly present in the flavylium form, respectively. At each $\mathrm{pH}$ value, the reaction medium was prepared by combining anthocyanins (malvidin-3-glucoside, cyanidin-3-glucoside, and peonidin-3-glucoside), (-)-epicatechin, and acetaldehyde in a molar ratio of $1: 6: 10$ at $25{ }^{\circ} \mathrm{C}$ in brown glass vials. The molar ratio of anthocyanins / (-)-epicatechin (1:6) used in this study corresponded to that in red wine, which is rich in these compounds [66]. Acetaldehyde, which was used as an excess reactant to provide ethyl linkage, was added to the reaction solution according to our previous procedure with slight modifications [67]. Malvidin-3-glcoside/(-)-epicatechin/acetaldehyde molar ratios of 1:3:10 and 1:1:10 were also studied to determine the conditions for optimal utilization of the reactants. The reaction between malvidin-3-glcoside and (-)-epicatechin was studied at reaction temperatures of 30, 35, and $40{ }^{\circ} \mathrm{C}$ in to determine the effect of temperature on the reaction rate. A total of 16 reaction systems were established. The reaction products were monitored periodically by HPLC-DAD and ESI-MS under the conditions described below. The optimized reaction conditions were determined from the reaction rates and the maximum yields of the reaction products. 


\subsection{HPLC-DAD Analysis}

The HPLC system was used to monitor the reaction products, equipped with a quaternary pump (Waters e2695), a controller (Waters e2695), an autosampler (Waters e2695), and a photodiode array detector (2998 PDA detector) coupled to a data processing computer (Empower ${ }^{\mathrm{TM}} 2$ chromatography data software). The column was Innoval C18 $(5 \mu \mathrm{m}, 4.6 \times 250 \mathrm{~mm})$ and the temperature was set at $30^{\circ} \mathrm{C}$. The flow rate of the mobile phase was fixed at $0.7 \mathrm{~mL} / \mathrm{min}$. Two elution solvents, A (water:formic acid; 98:2, $v / v$ ) and $B$ (water:acetonitrile:formic acid; 68:30:2, $v / v$ ), were used with the gradient elution program as follows: $0 \mathrm{~min}, 18 \% \mathrm{~B} ; 42-48 \mathrm{~min}, 47 \% \mathrm{~B} ; 78-110 \mathrm{~min}, 100 \% \mathrm{~B}$; and re-equilibration of the column for $10 \mathrm{~min}$. The detection wavelength was $525 \mathrm{~nm}$ for the detection of anthocyanins and their derivatives and $280 \mathrm{~nm}$ for all polyphenols.

\subsection{Isolation and Purification of Ethyl-Linked Anthocyanin-Flavanol Pigments}

Individual ethyl-linked anthocyanin-flavanol pigments were formed from each of the condensation reactions between anthocyanins and (-)-epicatechin mediated by acetaldehyde under the optimized conditions described above. When the maximal yields of the reaction products were reached in the reaction solution, the ethyl-linked anthocyanin-flavanol pigments were isolated and purified using a Shimadzu LC-20AR module equipped with an SPD-20AV detector coupled to a data processing computer (LabSolutions, Kyoto, Japan). The wavelength was set at $280 \mathrm{~nm}$. The column was YMC-Pack ODS-A $(250 \times 10 \mathrm{~mm}, 5 \mu \mathrm{m})$ and the temperature was kept at $30^{\circ} \mathrm{C}$. The flow rate of the mobile phase was fixed at $4.0 \mathrm{~mL} / \mathrm{min}$. To isolate individual ethyl-linked anthocyanin-flavanol pigments, gradient elution was performed with two solvents, A (water:formic acid; 98:2, $v / v$ ) and B (water:acetonitrile:formic acid; 68:30:2, v/v), as follows: $0 \mathrm{~min}, 18 \% \mathrm{~B} ; 15 \mathrm{~min}, 47 \% \mathrm{~B} ; 15-25 \mathrm{~min}, 47 \%$ B; $55 \mathrm{~min}, 100 \%$ B. For further purification of the ethyl-linked anthocyanin-flavanol pigments, isocratic elution was performed with two solvents, A (water:formic acid; 98:2, v/v) and B (acetonitrile), under chromatographic conditions of $12 \% \mathrm{~B}$.

\subsection{MS Analysis}

Identification of the compounds formed in the reaction solution was carried out by HPLC-ESI-QTOF-MS/MS (Agilent Technologies, Santa Clara, CA, USA) annlysis. MS/MS analysis was performed in positive ion mode using the following conditions: mass range recorded was from $\mathrm{m} / \mathrm{z}$ 50-1500; capillary voltage was $3500 \mathrm{~V}$; gas temperature was $300^{\circ} \mathrm{C}$; gas flow was $7 \mathrm{~L} / \mathrm{min}$; nebulizer pressure was $35 \mathrm{psi}$; sheath gas temperature was $325^{\circ} \mathrm{C}$; sheath gas flow $11 \mathrm{~L} / \mathrm{min}$.

\subsection{ECD Analysis}

\subsubsection{Circular Dichroism (CD) Spectra}

CD spectra were recorded in the range of 200-550 nm using a Bio-Logic MOS-450 spectrometer (Bio-Logic, Claix, France) at $25^{\circ} \mathrm{C}$. The path length of the quartz cuvette was $1 \mathrm{~cm}$. The sampling interval was set to $0.5 \mathrm{~s}$.

\subsubsection{Conformational Analysis}

Conformational analysis was initially performed using Confab [68] with the MMFF94 force field to systematically search for undetermined relative configurations ( $R$ and $S$ ) of cyanidin-3-glucoside-ethyl-epicatechin compounds. As the saccharide group was expected to have less of an effect on the ECD spectra, it was removed to simplify the structure in conformational analyses and ECD calculations. Room-temperature equilibrium populations were calculated according to the Boltzmann distribution law (Equation (1)). Conformers with a Boltzmann population over $0.01 \%$ were chosen for subsequent Quantum Mechanics (QM) calculations. 


$$
\frac{N_{i}}{N}=\frac{g_{i} e^{-\frac{E_{i}}{k_{B} T}}}{\sum g_{i} e^{-\frac{E_{i}}{k_{B} T}}}
$$

where $N_{i}$ is the number of conformer $\mathbf{i}$ with energy $E_{i}$ and degeneracy $g_{i}$ at temperature $\mathbf{T}$, and $k_{B}$ is Boltzmann constant.

\subsubsection{ECD Calculation}

The theoretical calculations were conducted using Gaussian 09 (Revision D.01. Gaussian Inc., Wallingford, CT, USA). Firstly, conformers were optimized at the PM6 level of theory using a semiempirical method. The conformers with a Boltzmann population lower than $1 \%$ were filtered and the remaining conformers were further optimized at the B3LYP/6-311G $(d, p)$ level of theory in methanol using the IEFPCM model. Vibrational frequency analysis confirmed the stable structures. Under the same conditions, the ECD calculation was conducted using time-dependent density functional theory (TD-DFT). Rotatory strengths for a total of 100 excited states were calculated. The ECD spectrum was simulated in SpecDis 1.64 [69] by overlapping Gaussian functions for each transition according to Equation (2).

$$
\Delta \varepsilon(\mathrm{E})=\frac{1}{2.297 \times 10^{-39}} \times \frac{1}{\sqrt{2 \pi \sigma}} \sum_{i}^{A} \Delta E_{i} R_{i} e^{-\left(\frac{E-E_{i}}{2 \sigma}\right)^{2}}
$$

where $\sigma$ represents the width of the band at $1 / e$ height, and $\Delta E_{i}$ and $R_{i}$ are the excitation energies and rotatory strengths for transition $\mathbf{i}$, respectively.

The $\sigma$ and UV-shift parameters were $0.32 \mathrm{eV}$ and $20 \mathrm{~nm}$, respectively, for $R$ configurations, and $0.32 \mathrm{eV}$ and $30 \mathrm{~nm}$, respectively, for $S$ configurations.

\subsection{Antioxidant Activity}

The in vitro antioxidant activities of (-)-epicatechin, anthocyanins (malvidin-3-glucoside, cyanidin-3-glucoside, and peonidin-3-glucoside), and ethyl-linked anthocyanin-flavanol pigments were analyzed by three common methods. DPPH and ABTS assays were implemented by measuring free-radical scavenging capacities, whereas the FRAP assay was conducted by evaluating ferric-reducing antioxidant power.

\subsubsection{DPPH Assay}

The DPPH assay has been considered a standard and easy colorimetric method for estimating antioxidant properties by assessing the free-radical scavenging capacities of the antioxidants. The scavenging abilities of the samples for DPPH were determined as described in our previous method with slight modifications [65]. Briefly, a DPPH solution was diluted with methanol to obtain an absorbance of $0.74( \pm 0.02)$ at $517 \mathrm{~nm}$. Then, $5 \mu \mathrm{L}$ of the sample solution or standard solution of Vc and Trolox at various concentrations and $200 \mu \mathrm{L}$ of DPPH solution were added to a 96-well microplate (Corning, NY, USA). The absorbance of the reaction mixture at $517 \mathrm{~nm}$ was recorded using a microplate reader (Tecan Infinite M200 Pro, Männedorf, Switzerland) at the steady state reached after $100 \mathrm{~min}$ of reaction at room temperature in the dark, using methanol as a blank reference. The scavenging ability for DPPH radicals was calculated using Equation (3):

$$
\text { Scavenging rate }(\%)=(\mathrm{A} 0-\mathrm{Ai}) \times 100
$$

where $\mathrm{A} 0$ and $\mathrm{Ai}$ are the absorbance of the control and the sample, respectively.

The antioxidant activity was expressed as EC50, defined as the amount of antioxidant needed to decrease the initial free-radical concentration by $50 \%$. The EC50 value can be obtained from the 
dose-response curve using regression analysis. The calculation was performed using SPSS software (Version 22.0, Chicago, IL, USA).

\subsubsection{ABTS Assay}

Antioxidant activities can be evaluated by measuring the ability to scavenge the ABTS. ${ }^{+}$free radical according to our previous method [65] with some modifications. An ABTS stock solution was obtained by dissolving ABTS in a phosphate buffered saline (PBS, pH 7.4) solution to a $7 \mathrm{mM}$ concentration. Equal amounts of ABTS stock solution and $2.45 \mathrm{mM}$ potassium persulfate were mixed and then allowed to react in the dark at room temperature for 12-16 $\mathrm{h}$ to produce ABTS radical cations (ABTS. ${ }^{+}$). The ABTS. ${ }^{+}$solution was then diluted with PBS to obtain an absorbance of $0.74 \pm 0.03$ at $734 \mathrm{~nm}$ before use. Then, $200 \mu \mathrm{L}$ of ABTS. ${ }^{+}$solution was added to each well of a 96-well microplate together with $10 \mu \mathrm{L}$ of sample solution or standard solution at various concentrations. After reacting at room temperature for $240 \mathrm{~min}$, the absorbance was recorded at $734 \mathrm{~nm}$ against a blank reference of PBS using microplate reader described above. The ABTS $\cdot^{+}$radical cation scavenging activity was calculated using Equation (3) and the EC50 value was obtained as described above.

\subsubsection{FRAP Assay}

The ferric-reducing antioxidant power (FRAP) assay, based on the reduction of $\mathrm{Fe}^{3+}$-TPTZ to blue-colored $\mathrm{Fe}^{2+}$-TPTZ, was conducted based on the procedure described in our previous work [65] with slight modifications. Briefly, the FRAP stock solution was composed of $300 \mathrm{mM}$ acetate buffer (pH 3.6), $10 \mathrm{mM}$ TPTZ solution in $40 \mathrm{mM} \mathrm{HCl}$, and $20 \mathrm{mM} \mathrm{FeCl}_{3} \cdot 6 \mathrm{H}_{2} \mathrm{O}$ solution. A fresh working solution was prepared by mixing these three solutions at a ratio of $10: 1: 1$ at $37^{\circ} \mathrm{C}$. Sample $(5 \mu \mathrm{L})$ or standard solutions at various concentrations were allowed to react with $180 \mu \mathrm{L}$ of the working solution at $37^{\circ} \mathrm{C}$ for $390 \mathrm{~min}$ in a 96 -well microplate. Then, the absorbance was recorded at $593 \mathrm{~nm}$, against a reagent blank. The FRAP value was expressed as $\mu \mathrm{M} \mathrm{FeSO}_{4} / \mu \mathrm{M}$ sample under the same absorbance.

\subsection{Statistical Analysis}

All experiments were performed in triplicate and results are expressed as means \pm standard deviation (SD). The comparison of means was determined by one-way analysis of variance (ANOVA) followed by Duncan's multiple range tests. Correlations among data obtained were calculated using Pearson's correlation coefficient (r). All statistical analyses were performed using SPSS (Version 22.0, Chicago, IL, USA).

\section{Conclusions}

Condensation reactions between three anthocyanins (malvidin-3-glucoside, cyanidin-3-glucoside, and peonidin-3-glucoside) and (-)-epicatechin mediated by acetaldehyde were implemented in a model wine solution. The optimized reaction conditions for ethyl-linked anthocyanin-flavanol pigments were obtained. Six individual ethyl-linked anthocyanin-flavanol pigments were isolated from the reaction mixture and their structures were identified by HPLC-QTOF-MS and ECD analyses. Furthermore, all six ethyl-linked anthocyanin-flavanol pigments showed higher antioxidant activities than anthocyanins, and the ethyl-linked anthocyanin-flavanol pigments containing cyanidin-3-glucoside unit exhibited the highest antioxidant properties. The effect of configuration on the antioxidant activities of the ethyl-linked anthocyanin-flavanol pigments was not significant.

Author Contributions: Conceived and designed the experiments: Lingxi Li and Baoshan Sun. Performed the experiments: Lingxi Li and Minna Zhang. Analysed the data: Lingxi Li, Minna Zhang and Shuting Zhang. Wrote or contributed to the writing of the manuscript: Lingxi Li, Baoshan Sun and Yan Cui.

Acknowledgments: This work was supported by Program for Liaoning Excellent Talents in University (LJQ2015106).

Conflicts of Interest: The authors declare no conflict of interest. 


\section{References}

1. Timberlake, C.F.; Bridle, P. Interactions between anthocyanins, phenolic compounds, and acetaldehyde and their significance in red wines. Am. J. Enol. Viticult. 1976, 27, 97-105.

2. Haslam, E. In vino veritas: Oligomeric procyanidins and the ageing of red wines. Phytochemistry 1980, 19, 2577-2582.

3. Casassa, L.F.; Keller, M.; Harbertson, J.F. Regulated deficit irrigation alters anthocyanins, tannins and sensory properties of cabernet sauvignon grapes and wines. Molecules 2015, 20, 7820-7844. [CrossRef] [PubMed]

4. Preys, S.; Mazerolles, G.; Courcoux, P.; Samson, A.; Fischer, U.; Hanafi, M.; Bertrand, D.; Cheynier, V. Relationship between polyphenolic composition and some sensory properties in red wines using multiway analyses. Anal. Chim. Acta 2006, 563, 126-136. [CrossRef]

5. Ricardo-da-Silva, J.M.; Darmon, N.; Fernandez, Y.; Mitjavila, S. Oxygen free radical scavenger capacity in aqueous models of different procyanidins from grape seeds. J. Agric. Food Chem. 1991, 39, 1549-1552. [CrossRef]

6. Zhao, G.; Gao, H.; Jie, Q.; Lu, W.; Wei, X. The molecular mechanism of protective effects of grape seed proanthocyanidin extract on reperfusion arrhythmias in rats in vivo. Biol. Pharm. Bull. 2010, 33, 759-767. [CrossRef] [PubMed]

7. Kruger, M.J.; Davies, N.; Myburgh, K.H.; Lecour, S. Proanthocyanidins, anthocyanins and cardiovascular diseases. Food Res. Int. 2014, 59, 41-52. [CrossRef]

8. Renaud, S.; de Lorgeril, M. Wine, alcohol, platelets, and the french paradox for coronary heart disease. Lancet 1992, 339, 1523-1526. [CrossRef]

9. Aviram, M.; Fuhrman, B. Polyphenolic flavonoids inhibit macrophage-mediated oxidation of LDL and attenuate atherogenesis. Atherosclerosis 1998, 137, S45-S50. [CrossRef]

10. Spranger, I.; Sun, B.; Mateus, A.M.; Freitas, V.D.; Ricardo-da-Silva, J.M. Chemical characterization and antioxidant activities of oligomeric and polymeric procyanidin fractions from grape seeds. Food Chem. 2008, 108, 519-532. [CrossRef] [PubMed]

11. Cueva, C.; Gil-Sanchez, I.; Ayuda-Duran, B.; Gonzalez-Manzano, S.; Gonzalez-Paramas, A.M.; Santos-Buelga, C.; Bartolome, B.; Moreno-Arribas, M.V. An integrated view of the effects of wine polyphenols and their relevant metabolites on gut and host health. Molecules 2017, 22, 99. [CrossRef] [PubMed]

12. Sun, B.; Leandro, M.C.; de Freitas, V.; Spranger, M.I. Fractionation of red wine polyphenols by solid phase extraction and liquid chromatography. J. Chromatogr. A 2006, 1128, 27-38. [CrossRef] [PubMed]

13. Boulton, R. The copigmentation of anthocyanins and its role in the color of red wine: A critical review. Am. J. Enol. Viticult. 2001, 52, 67-87.

14. Schwarz, M.; Picazo-Bacete, J.J.; Winterhalter, P.; Hermosín-Gutiérrez, I. Effect of copigments and grape cultivar on the color of red wines fermented after the addition of copigments. J. Agric. Food Chem. 2005, 53, 8372-8381. [CrossRef] [PubMed]

15. Nagel, C.W.; Wulf, L.W. Changes in the anthocyanins, flavonoids and hydroxycinnamic acid esters during fermentation and aging of merlot and cabernet sauvignon. Am. J. Enol. Viticult. 1979, 30, 111-116.

16. Somers, T.C.; Michael, E.E. Grape pigment phenomena: Interpretation of major colour losses during vinification. J. Sci. Food Agric. 1979, 30, 623-633. [CrossRef]

17. Es-Safi, N.E.; Cheynier, V.; Moutounet, M. Interactions between cyanidin 3-O-glucoside and furfural derivatives and their impact on food color changes. J. Agric. Food Chem. 2002, 50, 5586-5595. [CrossRef] [PubMed]

18. Eglinton, J.; Griesser, M.; Henschke, P.; Kwiatkowski, M.; Parker, M.; Herderich, M. Yeast-mediated formation of pigmented polymers in red wine. In Red Wine Color; American Chemical Society: Washington, DC, USA, 2004; Volume 886, pp. 7-21.

19. Es-Safi, N.E.; Fulcrand, H.; Cheynier, V.; Moutounet, M. Studies on the acetaldehyde-induced condensation of (-)-epicatechin and malvidin 3-O-glucoside in a model solution system. J. Agric. Food Chem. 1999, 47, 2096-2102. [CrossRef] [PubMed]

20. Dueñas, M.; Fulcrand, H.; Cheynier, V. Formation of anthocyanin-flavanol adducts in model solutions. Anal. Chim. Acta 2006, 563, 15-25. [CrossRef]

21. Salas, E.; Fulcrand, H.; Emmanuelle Meudec, A.; Cheynier, V. Reactions of anthocyanins and tannins in model solutions. J. Agric. Food Chem. 2003, 51, 7951. [CrossRef] [PubMed] 
22. Pissarra, J.; Mateus, N.; Rivas-Gonzalo, J.; Santos Buelga, C.; De Freitas, V. Reaction between malvidin 3-glucoside and (+)-catechin in model solutions containing different aldehydes. J. Food Sci. 2003, 68, 476-481. [CrossRef]

23. Drinkine, J.; Lopes, P.; Kennedy, J.A.; Teissedre, P.L.; Saucier, C. Ethylidene-bridged flavan-3-ols in red wine and correlation with wine age. J. Agric. Food Chem. 2007, 55, 6292-6299. [CrossRef] [PubMed]

24. Cheynier, V. Grape polyphenols and their reactions in wine. In Polyphenols; Martens, S., Treutter, T., Forkmann, G., Eds.; Freising-Weihenstephan: Bavaria, Germany, 2002; pp. 1-14.

25. Escott, C.; Del Fresno, J.M.; Loira, I.; Morata, A.; Tesfaye, W.; González, M.D.C.; Suárez-Lepe, J.A. Formation of polymeric pigments in red wines through sequential fermentation of flavanol-enriched musts with non-Saccharomyces yeasts. Food Chem. 2018, 239, 975-983. [CrossRef] [PubMed]

26. Bindon, K.A.; McCarthy, M.G.; Smith, P.A. Development of wine colour and non-bleachable pigments during the fermentation and ageing of (Vitis vinifera L. cv.) cabernet sauvignon wines differing in anthocyanin and tannin concentration. LWT Food Sci. Technol. 2014, 59, 923-932. [CrossRef]

27. Kunsági-Máté, S.; Szabó, K.; Nikfardjam, M.P.; Kollár, L. Determination of the thermodynamic parameters of the complex formation between malvidin-3-O-glucoside and polyphenols. Copigmentation effect in red wines. J. Biochem. biophys. Meth. 2006, 69, 113-119. [CrossRef] [PubMed]

28. Bindon, K.; Kassara, S.; Hayasaka, Y.; Schulkin, A.; Smith, P. Properties of wine polymeric pigments formed from anthocyanin and tannins differing in size distribution and subunit composition. J. Agric. Food Chem. 2014, 62, 11582-11593. [CrossRef] [PubMed]

29. Hayasaka, Y.; Birse, M.; Eglinton, J.; Herderich, M. The effect of saccharomyces cerevisiae and saccharomyces bayanus yeast on colour properties and pigment profiles of a cabernet sauvignon red wine. Aust. J. Grape Wine Res. 2007, 13, 176-185. [CrossRef]

30. Somers, T.C. The polymeric nature of wine pigments. Phytochemistry 1971, 10, 2175-2186. [CrossRef]

31. Sun, B.; Spranger, M.I. Changes in phenolic composition of tinta miúda red wines after 2 years of ageing in bottle: Effect of winemaking technologies. Eur. Food Res. Technol. 2005, 221, 305-312. [CrossRef]

32. Damianaki, A.; Bakogeorgou, E.; Kampa, M.; Notas, G.; Hatzoglou, A.; Panagiotou, S.; Gemetzi, C.; Kouroumalis, E.; Martin, P.M.; Castanas, E. Potent inhibitory action of red wine polyphenols on human breast cancer cells. J. Cell Biochem. 2000, 78, 429-441. [CrossRef]

33. Pinder, R.M.; Sandler, M. Alcohol, wine and mental health: Focus on dementia and stroke. J. Psychopharmacol. 2004, 18, 449-456. [CrossRef] [PubMed]

34. Guo, X.; Tresserra-Rimbau, A.; Estruch, R.; Martinez-Gonzalez, M.A.; Medina-Remon, A.; Castaner, O.; Corella, D.; Salas-Salvado, J.; Lamuela-Raventos, R.M. Effects of polyphenol, measured by a biomarker of total polyphenols in urine, on cardiovascular risk factors after a long-term follow-up in the predimed study. Oxid. Med. Cell. Longev. 2016, 2016, 2572606. [CrossRef] [PubMed]

35. Greyling, A.; Bruno, R.M.; Draijer, R.; Mulder, T.; Thijssen, D.H.J.; Taddei, S.; Virdis, A.; Ghiadoni, L. Effects of wine and grape polyphenols on blood pressure, endothelial function and sympathetic nervous system activity in treated hypertensive subjects. J. Funct. Foods 2016, 27, 448-460. [CrossRef]

36. Giovinazzo, G.; Grieco, F. Functional properties of grape and wine polyphenols. Plant Food. Hum. Nutr. 2015, 70, 454-462. [CrossRef] [PubMed]

37. German, J.B.; Walzem, R.L. The health benefits of wine. Annu. Rev. Nutr. 2000, 20, 561-593. [CrossRef] [PubMed]

38. Radovanovic, B.; Radovanovic, A. Free radical scavenging activity and anthocyanin profile of cabernet sauvignon wines from the balkan region. Molecules 2010, 15, 4213-4226. [CrossRef] [PubMed]

39. Cavallini, G.; Straniero, S.; Donati, A.; Bergamini, E. Resveratrol requires red wine polyphenols for optimum antioxidant activity. J. Nutr. Health Aging 2016, 20, 540-545. [CrossRef] [PubMed]

40. Vinayagam, R.; Jayachandran, M.; Xu, B. Antidiabetic effects of simple phenolic acids: A comprehensive review. Phytother. Res. 2016, 30, 184-199. [CrossRef] [PubMed]

41. Boam, T. Anti-androgenic effects of flavonols in prostate cancer. ecancermedicalscience 2015, 9, 585. [CrossRef] [PubMed]

42. Li, L.; Sun, B. Grape and wine polymeric polyphenols: Their importance in enology. Crit. Rev. Food Sci. 2017, 1-17. [CrossRef] [PubMed]

43. Brouillard, R. Flavonoids and flower colour. In The flavonoids; Harborne, J.B., Ed.; Chapman \& Hall: London, UK, 1988; pp. 525-538. 
44. Burtch, C.E.; Mansfield, A.K.; Manns, D.C. Reaction kinetics of monomeric anthocyanin conversion to polymeric pigments and their significance to color in interspecific hybrid wines. J. Agric. Food Chem. 2017, 65, 6379-6386. [CrossRef] [PubMed]

45. He, F.; Liang, N.N.; Mu, L.; Pan, Q.H.; Wang, J.; Reeves, M.J.; Duan, C.Q. Anthocyanins and their variation in red wines I. Monomeric anthocyanins and their color expression. Molecules 2012, 17, 1571-1601. [CrossRef] [PubMed]

46. Dipalmo, T.; Crupi, P.; Pati, S.; Clodoveo, M.; Luccia, A. Studying the evolution of anthocyanin-derived pigments in a typical red wine of southern Italy to assess its resistance to aging. LWT Food Sci. Technol. 2016, 71, 1-9. [CrossRef]

47. Sun, B.; Fernandes, T.A.; Spranger, M.I. A new class of anthocyanin-procyanidin condensation products detected in red wine by electrospray ionization multi-stage mass spectrometry analysis. Rapid Commun. Mass Spectrom. 2010, 24, 254-260. [CrossRef] [PubMed]

48. Boido, E.; Alcalde-Eon, C.; Carrau, F.; Dellacassa, E.; Rivas-Gonzalo, J.C. Aging effect on the pigment composition and color of Vitis vinifera L. cv. Tannat wines. Contribution of the main pigment families to wine color. J. Agric. Food Chem. 2006, 54, 6692-6704. [CrossRef] [PubMed]

49. Pati, S.; Losito, I.; Gambacorta, G.; La Notte, E.; Palmisano, F.; Zambonin, P.G. Simultaneous separation and identification of oligomeric procyanidins and anthocyanin-derived pigments in raw red wine by HPLC-UV-ESI-MSn. J. Mass Spectrom. 2006, 41, 861-871. [CrossRef] [PubMed]

50. Blanco-Vega, D.; Gomez-Alonso, S.; Hermosin-Gutierrez, I. Identification, content and distribution of anthocyanins and low molecular weight anthocyanin-derived pigments in Spanish commercial red wines. Food Chem. 2014, 158, 449-458. [CrossRef] [PubMed]

51. He, F.; Liang, N.N.; Mu, L.; Pan, Q.H.; Wang, J.; Reeves, M.J.; Duan, C.Q. Anthocyanins and their variation in red wines. II. Anthocyanin derived pigments and their color evolution. Molecules 2012, 17, 1483-1519. [CrossRef] [PubMed]

52. Nugroho, A.E.; Morita, H. Circular dichroism calculation for natural products. J. Nat. Med. 2014, 68, 1-10. [CrossRef] [PubMed]

53. Sun, B.; Neves, A.C.; Fernandes, T.A.; Fernandes, A.L.; Mateus, N.; De Freitas, V.; Leandro, C.; Spranger, M.I. Evolution of phenolic composition of red wine during vinification and storage and its contribution to wine sensory properties and antioxidant activity. J. Agric. Food Chem. 2011, 59, 6550-6557. [CrossRef] [PubMed]

54. Lingua, M.S.; Fabani, M.P.; Wunderlin, D.A.; Baroni, M.V. In vivo antioxidant activity of grape, pomace and wine from three red varieties grown in argentina: Its relationship to phenolic profile. J. Funct. Foods 2016, 20, 332-345. [CrossRef]

55. Dumitriu, D.; Peinado, R.A.; Peinado, J.; de Lerma, N.L. Grape pomace extract improves the in vitro and in vivo antioxidant properties of wines from sun light dried Pedro Ximénez grapes. J. Funct. Foods 2015, 17, 380-387. [CrossRef]

56. Li, D.; Zhang, Y.; Liu, Y.; Sun, R.; Xia, M. Purified anthocyanin supplementation reduces dyslipidemia, enhances antioxidant capacity, and prevents insulin resistance in diabetic patients. J. Nutr. 2015, 145, 742-748. [CrossRef] [PubMed]

57. Li, Y.; Li, L.; Cui, Y.; Zhang, S.; Sun, B. Separation and purification of polyphenols from red wine extracts using high speed counter current chromatography. J. Chromatogr. B 2017, 1054, 105-113. [CrossRef] [PubMed]

58. Kan, L.; Nie, S.-P.; Hu, J.; Liu, Z.; Xie, M. Antioxidant activities and anthocyanins composition of seed coats from twenty-six kidney bean cultivars. J. Funct. Foods 2016, 26, 622-631. [CrossRef]

59. Kahkonen, M.P.; Heinonen, M. Antioxidant activity of anthocyanins and their aglycons. J. Agric. Food Chem. 2003, 51, 628-633. [CrossRef] [PubMed]

60. Azevedo, J.; Oliveira, J.; Cruz, L.; Teixeira, N.; Bras, N.F.; De Freitas, V.; Mateus, N. Antioxidant features of red wine pyranoanthocyanins: Experimental and theoretical approaches. J. Agric. Food Chem. 2014, 62, 7002-7009. [CrossRef] [PubMed]

61. Muselík, J.; García-Alonso, M.P.; Martín-López, M.; Zemlicka, M.; Rivas-Gonzalo, J. Measurement of antioxidant activity of wine catechins, procyanidins, anthocyanins and pyranoanthocyanins. Int. J. Mol. Sci. 2007, 8, 797-809. [CrossRef]

62. Shon, M.Y.; Lee, J.; Choi, J.H.; Choi, S.Y.; Nam, S.H.; Seo, K.I.; Lee, S.W.; Sung, N.J.; Park, S.K. Antioxidant and free radical scavenging activity of methanol extract of chungkukjang. J. Food Compos. Anal. 2007, 20, 113-118. [CrossRef] 
63. Azevedo, J.; Fernandes, I.; Faria, A.; Oliveira, J.; Fernandes, A.; de Freitas, V.; Mateus, N. Antioxidant properties of anthocyanidins, anthocyanidin-3-glucosides and respective portisins. Food Chem. 2010, 119, 518-523. [CrossRef]

64. Ali, H.M.; Almagribi, W.; Al-Rashidi, M.N. Antiradical and reductant activities of anthocyanidins and anthocyanins, structure-activity relationship and synthesis. Food Chem. 2016, 194, 1275-1282. [CrossRef] [PubMed]

65. Li, L.X.; Zhang, S.T.; Cui, Y.; Li, Y.Y.; Luo, L.X.; Zhou, P.Y.; Sun, B.S. Preparative separation of cacao bean procyanidins by high-speed counter-current chromatography. J. Chromatogr. B 2016, 1036, 10-19. [CrossRef] [PubMed]

66. Sun, B.; Santos, C.P.; Leandro, M.C.; De Freitas, V.; Spranger, M.I. High-performance liquid chromatography/electrospray ionization mass spectrometric characterization of new products formed by the reaction between flavanols and malvidin 3-glucoside in the presence of acetaldehyde. Rapid Commun. Mass Spectrom. 2007, 21, 2227-2236. [CrossRef] [PubMed]

67. Sun, B.; Barradas, T.; Leandro, C.; Santos, C.; Spranger, I. Formation of new stable pigments from condensation reaction between malvidin 3-glucoside and (-)-epicatechin mediated by acetaldehyde: Effect of tartaric acid concentration. Food Chem. 2008, 110, 344-351. [CrossRef] [PubMed]

68. O’Boyle, N.M.; Vandermeersch, T.; Flynn, C.J.; Maguire, A.R.; Hutchison, G.R. Confab-Systematic generation of diverse low-energy conformers. J. Cheminform. 2011, 3, 8. [CrossRef] [PubMed]

69. Bruhn, T.; Schaumloffel, A.; Hemberger, Y.; Bringmann, G. Specdis: Quantifying the comparison of calculated and experimental electronic circular dichroism spectra. Chirality 2013, 25, 243-249. [CrossRef] [PubMed]

Sample Availability: Samples of the compounds are not available from the authors.

(C) 2018 by the authors. Licensee MDPI, Basel, Switzerland. This article is an open access article distributed under the terms and conditions of the Creative Commons Attribution (CC BY) license (http:/ / creativecommons.org/licenses/by/4.0/). 